\title{
Joint Routing and Congestion Control in Multipath Channel based on Signal to Noise Ratio with Cross Layer Scheme
}

\author{
Istikmal $^{1}$, Adit Kurniawan ${ }^{2}$, Hendrawan $^{3}$ \\ ${ }^{1,2,3}$ School of Electrical Engineering and Informatics, Institut Teknologi Bandung, Indonesia \\ ${ }^{1}$ School of Electrical Engineering, Telkom University, Indonesia
}

\begin{tabular}{l} 
Article Info \\
\hline Article history: \\
Received Nov 4, 20 \\
Revised Jan 15, 2018 \\
Accepted Mar 30, 201 \\
\hline Keyword: \\
Congestion control \\
Cross layer \\
Rayleigh \\
Rician \\
Routing \\
SNR \\
TCP
\end{tabular}

\section{Corresponding Author:}

Istikmal,

School of Electrical Engineering, Telkom University,

Jl. Telekomunikasi no 1 Terusan Buah Batu Bandung 40257 Indonesia.

Email: istikmal@telkomuniversity.ac.id

\begin{abstract}
Routing protocol and congestion control in Transmission Control Protocol (TCP) have important roles in wireless mobile network performance. In wireless communication, the stability of the path and successful data transmission will be influenced by the channel condition. This channel condition constraints come from path loss and the multipath channel fading. With these constraints, the algorithm in the routing protocol and congestion control is confronted with the uncertainty of connection quality and probability of successful packet transmission, respectively. It is important to investigate the reliability and robustness of routing protocol and congestion control algorithms in dealing with such situation. In this paper, we develop a detailed approach and analytical throughput performance with a cross layer scheme (CLS) between routing and congestion control mechanism based on signal to noise ratio (SNR) in Rician and Rayleigh as multipath fading channel. We proposed joint routing and congestion control TCP with a cross layer scheme model based on SNR (RTCP-SNR). We compare the performance of RTCP-SNR with conventional routing-TCP and routing-TCP that used CLS with routing aware (RTCP-RA) model. The analyses and the simulation results showed that RTCP-SNR in a multipath channel outperforms conventional routing-TCP and RTCP-RA.
\end{abstract}

Copyright $(2018$ Institute of Advanced Engineering and Science. All rights reserved.

\section{INTRODUCTION}

Wireless mobile ad hoc networks are dynamic networks in which devices are free to move and connect each other. This capability has a great impact on routing protocol and congestion control TCP strategy. The important issues of the routing protocol and TCP are the performance degradation and low adaptation in the wireless mobile adhoc network. Connection failures, route change and misperception of congestion control mechanism may caused TCP to inaccurately assume that all packet losses are due to network congestion which has been the main cause to network performance degradation. The cross layer scheme has become one of most promising methods to solve this problem [1]. This method shares information between layers and joint cooperations to achieve maximum network utility performance with general or specific solutions [2], [3].

Recently, cross layer design with involved routing and congestion control in TCP was present. Link quality and congestion awareness with a cross layer method were investigated in [4] for wireless routing obtained from MAC (Medium Access Control)-layer. A cross layer design for joint congestion control, routing, and scheduling was proposed in [5] by exploiting physical layer techniques. Another cross layer scheme for joint routing, scheduling, and congestion control was evaluated in [6] through network utility 
maximization (NUM). TCP-aware source routing (TSR) was proposed in [7], which minimizes TCP's consecutive timeouts by reducing the number of invalid in DSR (Dynamic Source Route) route. While [8], proposed the cross-layer between the MAC and network layers lead to early detection of congestion by routing aware. In [9], the cross layer approach was proposed to optimize the TCP throughput with cooperative relaying network. However, in previous study, routing and TCP with CLS were developed without congestion control adjustment at current congestion window when misperception occur and used the conventional congestion control mechanism. For example, [7]-[9] used routing aware by sending the data through the alternative route, when the network has a problem with invalid route, congestion, buffer overflow or relay condition, thus we call this CLS model as routing-TCP with routing aware (RTCP-RA). Unfortunately, wireless network not only suffer from the random network condition, but also from random variations in channel condition, that lead to poor performance [10], especially in multipath fading, such as Rician and Rayleigh environment.

This channel condition constraint occurs due to path loss and the multipath channel fading. With this constraint, the algorithm in the routing protocol and congestion control have to deal with the uncertainty connection quality and probability of successful packet transmission. In this situation, channel fadings produce high path break and bit error rate (BER), and lead the routing protocol and TCP to change the route frequently and enter a loss indication by packet loss and time-out that are not necessarily related to network congestion, respectively. It is important to investigate the reliability and robustness of routing protocol and congestion control algorithms in the dealing with this situation. Therefore, in this paper, that becomes our contributions, first, we develop a detailed approach and analytical throughput performance with a cross layer scheme between routing and congestion control mechanism based on signal to noise ratio (SNR) in Rician and Rayleigh environment. Second, based on this analytical method, we proposed joint mechanism between routing and congestion control in TCP based on SNR (RTCP-SNR). This proposed mechanism employed a cross layer scheme model by exploiting physical layer to be used in routing protocol mechanism as the routing metric to find the best path and to adjust congestion window at congestion control in TCP.

The paper is organized as follows. Section 2 provides an overview of the mechanism of routing protocol and congestion control in TCP. Section 3 describes the multipath channel propagation model. Section 4 explains the analytical throughput performance in multipath fading and the proposed method. Section 5 shows the performance results compare with the theory and another method. Finally, Section 6 is the conclusion and future research opportunities.

\section{ROUTING PROTOCOL AND CONGESTION CONTROL IN TCP}

\subsection{Routing protocol}

Routing is the important concept in wired and wireless network for packet transmission [11] and to maximize the packet successfully passed to the destination node [12]. A routing protocol in a mobile ad hoc network has an important role in finding the data transmission path. In a wireless ad hoc network, the routing protocol can be classified into flat routing, hierarchical routing, and geographic routing [13]. The flat routing is categorized based on proactive and reactive mechanism in finding the path. In this paper, we used a flat routing protocol with reactive mechanism as the role model, such as adhoc on-demand distance vector (AODV) [14] routing protocol. In the reactive routing protocol, the process of the route search is started when the source wants to send the data.

Generally, a flat reactive routing protocol uses minimum hop count as the routing metric to find the path. Figure 1 shows the routing protocol mechanism model in adhoc network, where $\mathrm{S}$ is the source, D as the destination, and IN is the intermediate node. With minimum hop count, routing protocol select S-(IN-3)-D as the path to delivery the data. In a mobile adhoc network, especially in a multipath fading environment, these routing protocols cannot work and adapt well, and usually show significant network performance degradation. In multipath fading environment, such as Rician and Rayleigh fading, routing protocols face uncertainty in fluctuation connection channel quality. When the routing protocol uses minimum hop count, the path will consist the connection with maximum range transmission to get the minimum hop path. This connection consequently has a low SNR channel quality. If the signal received by the receiver varies rapidly and the superposition of overall amount of the reflected signal, then this connection with low SNR will be weak and often disconnected.

Therefore, in this research, we propose routing protocol mechanism model with a cross layer scheme based on SNR. This routing protocol is channel aware that uses SNR threshold as a routing metric to find the best path. This SNR threshold is calculated based on maximum and the average SNR connection in a path. To gain higher advantage toward a more stable connectivity, we also propose joint TCP congestion control mechanism model with the routing protocol. The detail method is described in Section 4. 


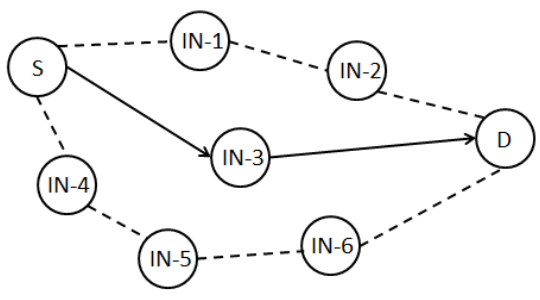

Figure 1. Routing protocol mechanism model in ad-hoc network

\subsection{Congestion control in TCP}

Transmission Control Protocol is a component of the transport layer. TCP provides reliable packet delivery services, which is end-to-end communication and connection oriented. There are currently a variety of data communication applications that useTCP as their transport protocol. TCP is widely used and developed, including in the ad-hoc network, but still requires evaluation and adjustment on the ad-hoc network characteristics [10], especially in a realistic environment model such as in Rician and Rayleigh with multipath fading.

TCP has a congestion control mechanism. The main objective in the TCP congestion control mechanism is to avoid congestion, which potentially decreases the effectiveness of the network. This mechanism aims to control the dynamic window size in accordance with the level of congestion on the network. Nearly all TCP versions assume that packets losses are due to congestion in the network. In this study, we applied TCP model that used loss based congestion control technique [15]. When a packet is detected to be lost, either by timeout (TO) or by triple-duplicate (TD) acknowledgment (ACK), TCP slows down the sending rate by adjusting its congestion window size. In the case that another time-out occurs before successfully retransmitting the packets lost during the first time out, the period of TO doubles until maximum 64TO is reached. Unfortunately, wireless networks suffer from several types of losses that are not related to congestion, making TCP cannot adapt well to this environment. This happens because the bit error rate in mobile adhoc network is high that cause packet loss, especially in case of severe fading and multipath, such as in Rician and Rayleigh. This misperception results in low performance of the networks.

Therefore, in our study, we proposed joint control mechanism with the routing protocol. When the routing protocol used SNR threshold as the routing metric, and a loss indication occurs due to channel quality degradation, we adjust the current congestion window based on SNR. The detailed approach described in Section 4.

\section{MULTIPATH CHANNEL PROPAGATION MODEL}

Receive signal power in the destination node can be calculated by propagation model. Propagation model can classify into large-scale and small-scale (fading) [16], while in implementation it can also divided into deterministic and probabilistic models. In the large-scale model, the path loss is the main component of a propagation model as a function of distance and other factors. Small-scale fading refers to the dramatic changes in the envelope of the received signal. These changes in the signal amplitude and phase are described statistically by a stochastic process.

Probabilistic model is a more realistic propagation model. For each transmission, the received power is described in the distribution. This leads to more varied results obtained from the successful reception. This happens with different probabilities as two adjacent users cannot communicate. The opposite can occur of probability of two users can communicate outside the range deterministic transmission. Distribution of this effect depends on probability models and parameters used. Rician and Rayleigh are probabilistic models. Model of Rician distribution takes into account the effects of a line of sight (LOS) path with a certain scale factor. The Ricean distribution is given by [16] as:

$$
p(z)=\left\{\begin{array}{cc}
\frac{z}{\sigma^{2}} \exp \left[-\frac{z^{2}+A^{2}}{2 \sigma^{2}}\right] I_{0}\left(\frac{z A}{\sigma^{2}}\right) & z \geq 0, A \geq 0 \\
0 & z<0
\end{array}\right.
$$

where $z$ is the amplitude of the envelope of the received signal, $A^{2}$ is the peak amplitude of the dominant signal or the power in LOS components, and $2 \sigma^{2}$ is the average power in non-LOS multipath components. Denote $I_{0}$ as the modified Bessel function of 0th order. Where the average received power in this distribution 
can be calculated by:

$$
P_{r}=\int_{0}^{\infty} Z^{2} p(z) d x=A^{2}+2 \sigma^{2}
$$

Rician distribution is usually described in term of a fading parameter $K$, which is the ratio between the deterministic signal power and the power non-LOS multipath components, and is defined by

$$
K=\frac{A^{2}}{2 \sigma^{2}}
$$

Factor $K$ shows the severity of the fading; where large value implies more mild fading, and small value implies severe fading. The Rician distribution model can be used to predict the situation where multipath and LOS component co-occur. When the deterministic signal power (LOS component) becomes weaker or $K=0$, a channel with no LOS components and only multipath will be in Rayleigh distribution. The probability density function of the Rayleigh distribution given by [17] is writren as:

$$
p(z)\left\{\begin{array}{lr}
\frac{z}{\sigma^{2}} \exp \left[-\frac{z^{2}}{2 \sigma^{2}}\right] & 0 \leq z \leq \infty \\
0 & z<0
\end{array}\right.
$$

The signals received by the recipient are a superposition of the overall amount of the reflected signal due to multipath. This causes the signal received by the receiver will vary rapidly. This situation is suitable in mobile to mobile communication, such as a device to device communication. The envelope received signal can be described in the statistical time varying of a flat fading signal. Figure 2 shows the bit error rate (BER) performance of BPSK in Rician, Rayleigh, and AWGN fading channel, where Rician parameter $K=4$. We observe that the Rayleigh fading has the worst bit error rate (BER).

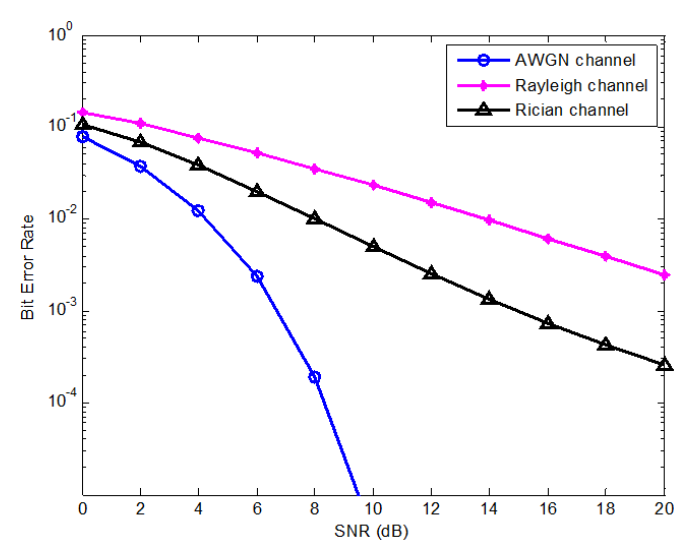

Figure 2. BER performance of BPSK in Rician, Rayleigh, and AWGN fading channels

\section{ANALYTICAL THROUGHPUT PERFORMANCE AND THE PROPOSED METHOD RTCP-SNR}

In this section, we develop analytical routing and congestion control mechanims through throughput performance formulation. Network throughput performance depends on the average number of successful data transmitted during the observation interval. If the average number of packets that successfully transmitted is $E[D]$, and the average duration of time required to transmit the data is $E[T]$, then we can calculate the throughput $B_{t h r}$ by:

$$
B_{t h r}=\frac{E[D]}{E[T]}
$$

The average propagation time is $t_{\text {prop }}$ and the process average time at all nodes is $t_{\text {proc }}$. If $\bar{C}$ is the average data transferred, with the average rate for delivery of the data is $R$, then the total average time to send $\bar{C}$ is $\bar{C} / R$ seconds. 
The average time to transmit the data $E[T]$ can be calculated as:

$$
E[T]=\frac{\bar{C}}{R}+2\left(t_{\text {proc }}+t_{\text {prop }}\right)
$$

Since the quality of channel link and the mobility of nodes will effect on the probability of bit error, this probability of bit error is influenced by the probability of success in a connection to transmit the data. Let $P_{r}$ is the average probability of packets successfully delivered in the transmitted data through the path. With this approach we can rewrite the $E[D]$ by:

$$
E[D]=\bar{C} P_{r}
$$

while the throughput as a function of $P_{r}$ and $R$ can be written as:

$$
B_{t h r}=\frac{\bar{C} P_{r}}{\bar{C}+2 R\left(t_{\text {proc }}+t_{\text {prop }}\right)} R \text {. }
$$

In this study, we used a stochastic model of TCP congestion control mechanism that yields a relatively simple analytic expression for the number of packets sent in the period [18], where TCP's behavior is presented in packet loss approach. This model is based on TCP that uses triple-duplicate (TD) ACK and timeout (TO) in congestion control, such as TCP Reno. Considering TD period (TDP) to be a period between two TD loss indications and timeout lost indication, this approach has been widely used. Based on this mechanism approach, the average data transferred is calculated based on probability of packet error or packet error rate, defined by:

$$
\bar{C}=E[\varphi]+E[\omega]-1+\delta E[R]
$$

$E[\varphi]$ is the average number of packets sent in a TD period up to and including the first packet that is lost. $E[\omega]$ is the average size of the TCP congestion window in packets. Denote $\delta$ is the probability that a loss indication ending a TDP is a TO, and $E[R]$ is the average number of packets sent during the time-out sequence. By considering loss indications are TD and TO, let $\varphi_{i}$ denotes the number of packets sent in a TD period up to and including the first packet that is lost. If the probability that $\varphi_{i}=k$ is the same to the probability that exactly $k-1$ packets are acknowledged before a loss occurs, we can calculate $E[\varphi]$ as:

$$
E[\varphi]=\sum_{k=1}^{\infty}(1-p)^{k-1} p k=\frac{1}{p^{\prime}}
$$

and the average size of TCP congestion window expressed by:

$$
E[\omega]=\frac{2+m}{3 m}+\sqrt{\frac{8(1-p)}{3 m p}+\left(\frac{2+m}{3 m}\right)^{2}},
$$

Further, a minimum probability that a loss indication ending a TDP is a TO with average number of packets sent during TO sequence is:

$$
\delta E[R]=\min \left(1, \frac{3}{c_{w}}\right) \frac{1}{(1-p)}
$$

where the number of packets acknowledged is $m$, and $c_{w}$ is the current congestion window size in packets. $p$ is the probability of packet error. With Equations (10)-(12), we can find the number of packets sent based on congestion control mechanism in Equation (9), so we can rewrite Equation (8) as:

$$
B_{t h r}=\frac{(E[\varphi]+E[\omega]-1+\delta E[R]) P_{r}}{E[\varphi]+E[\omega]-1+\delta E[R]+2 R\left(t_{\text {proc }}+t_{\text {prop }}\right)} R
$$

If $p_{i}$ is the probability of packet error on connection $i$, then the probability of success transmitting data on a connection $i$ is $\left(1-p_{i}\right)$. The total probability of success in sending the data for a path delivery that consists of a $N$ links connection can be written as follows:

$$
P_{r}=\prod_{i=1}^{N}\left(1-p_{i}\right)
$$


To analyze the correlation between the quality of the channel SNR with the throughput performance, we use the probability of error as Bit Error Rate (BER) function [19], which is given by:

$$
p=1-(1-\beta)^{L}
$$

where $\beta$ is the bit error rate and $L$ is the number of bits. We can calculate BER based on SNR and modulation technique. The probability of bit error often referred to as BER is a better performance measure to evaluate a modulation scheme. In this study, Binary Phase Shift Keying (BPSK) modulation is used. With this approach we can find the number of packets sent based on probability of error with congestion control and the probability of error in path connection as routing protocol decisions.

Next, to find the correlations between throughput performance with routing and congestion control that influence by the fading channel, we calculated the probability of error based on SNR in Rician and Rayleigh fading channel. In Rician fading channel, we used the probability of error as a BER function in [20], [21] and is given as:

$$
\bar{\beta}_{\text {Rician }}=Q_{1}(\vartheta, \mu)-\frac{1}{2}\left[1+\sqrt{\frac{d}{d+1}}\right] G,
$$

with $Q_{1}(\vartheta, \mu)$ is the Marcum $Q$ function defined as:

$$
\begin{aligned}
& Q_{1}(\vartheta, \mu)=\exp \left(-\frac{\vartheta^{2}+\mu^{2}}{2}\right) \sum_{l=0}^{\infty}\left(\frac{\vartheta}{\mu}\right)^{l} I_{o}(\vartheta \mu), \mu \geq \vartheta>0 \\
& Q_{1}(\vartheta, \mu)=Q(\vartheta-\mu), \mu \gg 1 \text { and } \mu \gg \mu-\vartheta
\end{aligned}
$$

and

$$
G=\exp \left(-\frac{\vartheta^{2}+\mu^{2}}{2}\right) I_{o}(\vartheta \mu)
$$

where

$$
\begin{aligned}
& \vartheta=\left[\sqrt{\left.\frac{K^{2}[1+2 d-2 \sqrt{d(d+1)}]}{2(d+1)}\right]},\right. \\
& \mu=\left[\sqrt{\left.\frac{K^{2}[1+2 d+2 \sqrt{d(d+1)}]}{2(d+1)}\right]}\right]
\end{aligned}
$$

The parameter $K$ is the Rician factor as described in Equation (3), $I_{o}(x)$ is the modified Bessel function of zero order and SNR is represented in $d=\sigma^{2} \frac{E_{b}}{N_{o}}$. Where, $\frac{E_{b}}{N_{o}}$ is the ratio of bit energy to noise power density. Based on Equations (15) and (16), we can calculate the average probability of error in Rician channel fading with BPSK modulation as:

$$
p_{\text {Rician }}=1-\left(1-Q_{1}(\vartheta, \mu)+\frac{1}{2}\left[1+\sqrt{\frac{d}{d+1}}\right] G\right)^{L}
$$

To evaluate a modulation scheme, usually the probability of bit error referred as BER [22]. In a Rayleigh fading channel, the average probability of error [17], [20] can be calculated by the following integral.

$$
\bar{\beta}=\int_{0}^{\infty} p_{\mathrm{AWGN}}(\gamma) p_{\gamma}(\gamma) d \gamma
$$

where $p_{\text {AWGN }}(\gamma)$ is the probability of error in additive white Gaussian noise (AWGN) at specific $\operatorname{SNR}(\gamma)$ and $p_{\gamma}(\gamma)$ is the probability density function of $\operatorname{SNR}(\gamma)$ due to the fading channel. For coherent detection of 
BPSK, we can calculate BER of BPSK modulation in AWGN channel byBER $=Q\left(\sqrt{2 E_{b} / N_{o}}\right) \approx Q(\sqrt{\gamma})$, where $E_{b} / N_{o}$ is the ratio of bit energy to noise power density. In Rayleigh fading channels, $\operatorname{SNR}(\gamma)=h^{2} \frac{E_{b}}{N_{o}}$, with the random variable $h$ is the channel gain, and $h^{2}$ represents the instantaneous power of the fading channel. The probability density function $p_{\gamma}(\gamma)$ given by [17], [22] is written as:

$$
p_{\gamma}(\gamma)=\frac{1}{\bar{\gamma}} \exp \left(-\frac{\gamma}{\bar{\gamma}}\right)
$$

where $\bar{\gamma}=\frac{E_{b}}{N_{o}} E\left[h^{2}\right]$ is the average signal-to-noise ratio, and the random variable $h^{2}$ represents the instantaneous power of the fading channel. Now, we can rewrite Equation (21) or Rayleigh fading channel by:

$$
\bar{\beta}_{\text {Rayleigh }}=\int_{0}^{\infty} Q(\sqrt{\gamma}) \frac{1}{\bar{\gamma}} e^{-\gamma / \bar{\gamma}} d \gamma
$$

By integrating $p \operatorname{AWGN}(\gamma)$ and $p_{f}(\gamma)$ in Equation (23) and with mathematical approach, we can find the average probability of error in Rayleigh fading with BPSK modulation as:

$$
\bar{\beta}_{\text {Rayleigh }}=\frac{1}{2}\left[1-\sqrt{\frac{\frac{\bar{\gamma}}{2}}{1+\frac{\gamma}{2}}}\right]
$$

Then, with Equation (15) and Equation (24), we can rewrite the packet error rate base on the average probability of error in Rayleigh fading and SNR, defined by:

$$
p_{\text {Rayleigh }}=1-\left(1-\frac{1}{2}\left[1-\sqrt{\frac{\bar{\gamma}}{1+\frac{\bar{\gamma}}{2}}}\right]\right)^{L}
$$

Probability of packet error will decrease with increased average SNR and smaller number of bits as shown in Figure 3. Now, with Equations (20) and (25), we can recalculate Equation (13) to find throughput performance with routing and congestion control mechanism based on SNR with a cross layer scheme in Rician and Rayleigh fading channels. Figure 4 shows higher average throughput of the network with increasing average SNR in the network connection. From Figure 3 and Figure 4, we observe that it is important to used physical layer in term of SNR as channel condition in routing protocol and congestion control algorithm to face channel fading environment.

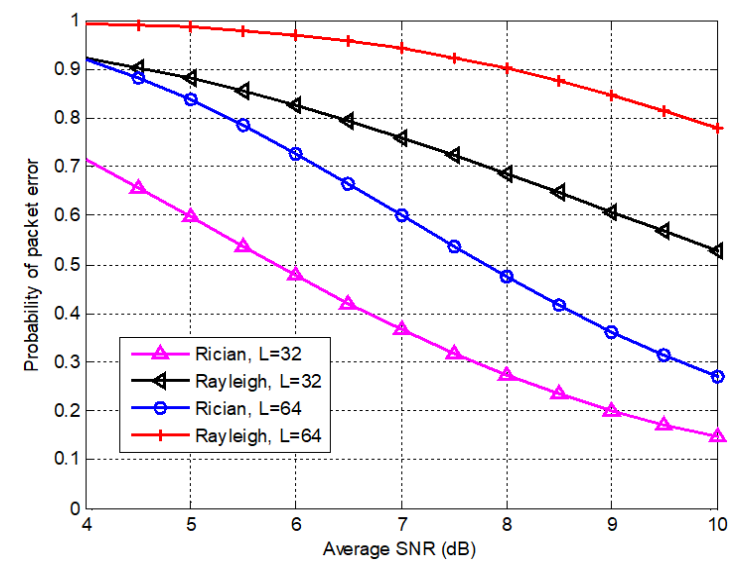

Figure 3. Probability of packet error with average SNR in Rician and Rayleigh fading channels

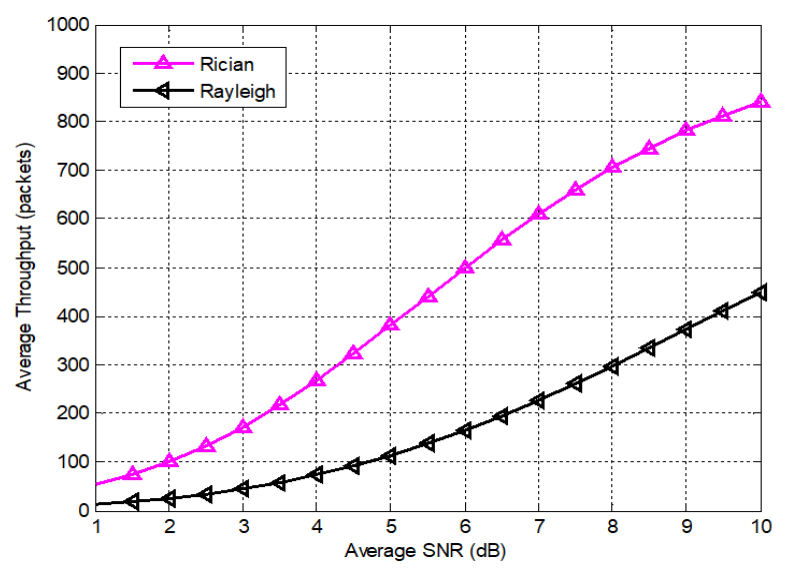

Figure 4. Average throughput with average SNR in Rician and Rayleigh fading channels 
Based on this analytical model, we proposed joint routing and congestion control in TCP based on SNR with cross layer scheme, namely Routing-TCP-SNR (RTCP-SNR). Figure 5 shows the cross layer framework of our proposed method. In this scheme model, routing protocol will select the path based on SNR threshold $\left(\gamma_{t h}\right)$ to get a higher average data transfer with congestion control in TCP mechanism and to avoid low channel quality connection, as described in Equation (13). In our proposed method, the SNR threshold as the routing metric is calculated by:

$$
\gamma_{t h}=\frac{\sum_{j=1}^{H_{i}} \gamma_{i, j}}{H_{i}}-\left(\max \left(\gamma_{i, j}\right)-\frac{\sum_{j=1}^{H_{i}} \gamma_{i, j}}{H_{i}}\right)
$$

where $\gamma_{i, j}$ is SNR in the path $i$ at link number $j$ and $H_{i}$ is the number of links or hops in path $i$. Maximum SNR or highest SNR connection in path $i$ at link number $j$ is defined as $\max \left(\gamma_{i, j}\right)$. Denote $\tau=\max \left(\gamma_{i, j}\right)-\left(\sum_{j=1}^{H_{i}} \gamma_{i, j} / H_{i}\right)$ as reduction factor of SNR threshold $\left(\gamma_{t h}\right)$, has the maximum value $\bar{\gamma}_{l} / 4$. For example, if the calculation of $\tau$ exceeding the $\sum_{j=1}^{H_{i}} \gamma_{i, j} / H_{i}$ then the reduction factor is set to $\bar{\gamma}_{l} / 4$. The path $i$ can be selected as the path to deliver the data $\left(\Gamma_{\text {path }}\right)$ when the path has highest SNR threshold $\max \left(\gamma_{t h}\right)$ and all the connection in the path has complied with the SNR threshold, which is:

$$
\Gamma_{p a t h}=\left\{\begin{array}{l}
\max \left(\gamma_{t h}\right)_{i} \\
\gamma_{i, j} \geq \gamma_{t h}
\end{array}\right.
$$

with this method, routing protocol in RTCP-SNR can select the path as the route with highest channel quality and avoid the connection with low SNR channel quality. While in RTCP-RA, although the algorithm can select path with good channel quality, but without SNR threshold in selecting a link as the path, one or more links can have a very low channel quality. With this issues, RTCP-RA may face path break, and this frequently occurs in multipath fading environment. This situation will lead to network performance decrease in RTCP-RA.

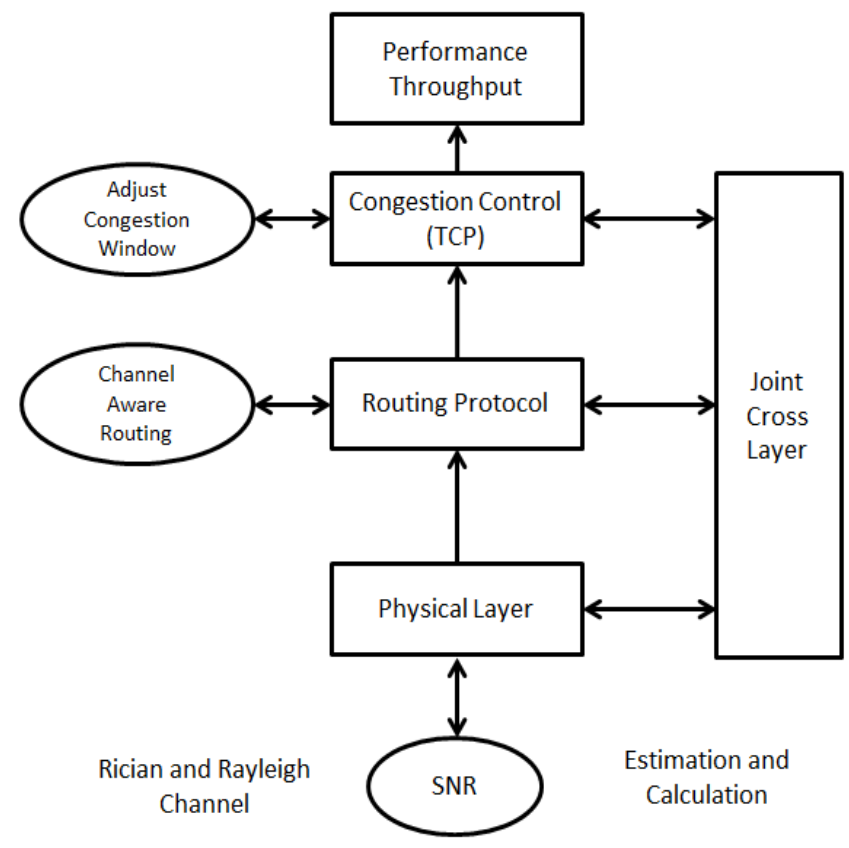

Figure 5. Proposed cross-layer framework

The issues in previous method in RTCP-RA model are present when the route has a problem with invalid route, buffer overflow, or relay condition, this method find the alternative route or relay to gain higher performance without congestion control adjustment in TCP. Although it seems like a more simplified CLS 
mechanism, it does not work as expected in multipath environment such as Rician and Rayleigh. In Rician and Rayleigh multipath channel, packet loss and route change may occur in high rate and lead to a loss indication in congestion control. Beside packet loss or TD as congestion indication, the route change may cause TOs that also indicate network congestion. When the method only considers alternative route, this can cost more time in re-route mechanism and cause TO. The majority of the network performance degradation due to TO than TD [18], and congestion control in TCP has misinterpretation about network condition. In such situation, packet loss mostly indicted as network congestion indication, meanwhile in Rician and Rayleigh packet loss occurs due to high bit error rate. This misperception makes congestion control which reduces the congestion window to one and doubles the time out period as the result network performance degradation. For that reason, we proposed congestion control model that adjust congestion window based on $\mathrm{SNR}$. If the channel condition is deteriorating and a loss indication occurs, then the current congestion window size $\left(c_{w}\right)$ in TCP can be adjusted based on SNR.

The minimum $c_{w}$ in TDP is 3 [18]. When this situation happen, we assume the signal vary rapidly that cause channel quality fluctuation on forward and backward data transmission mechanism. This severe fading and multipath can cause SNR degradation, and increase BER that cause packet or ACK loss and not by network congestion. Based on this approach, we define $E[\sigma]$ as the average number of packets sent in a TD period and the average number of packets sent during TO sequence based on minimum probability of a loss indication that ends a TDP is a TO, given by:

$$
E[\sigma]=\left\{\begin{array}{c}
\frac{3 p+c_{w}(1-p)}{c_{w} p(1-p)}, c_{w}>3 \\
\frac{1}{p(1-p)}, c_{w} \leq 3
\end{array}\right.
$$

By this proposed method, the congestion window can be reduced when the SNR connection has channel quality degradation, and increase the average congestion window when the probability packet error get smaller by keeping up the current congestion window in Equation (28). TCP can get higher $E[\sigma]$, especially in the minimum probability a loss indication ends a TDP is a TO and prevent the congestion window is reduced to one and a period of time out get doubled by the congestion control mechanism. The current congestion window can be adjusted according to channel condition, SNR, represented as the probability of error $(p)$ as described in Equations (20), (25), and (28). With this joint routing and congestion control mechanism with a CLS based on SNR, we can improve network performance.

\section{RESULTS AND ANALYSIS}

We evaluated conventional routing and TCP performance with congestion control based on TD and TO model, which is routing-TCP, and compare with our proposed method, routing-TCP-SNR (RTCP-SNR), that used cross layer scheme for joint routing and congestion control based on SNR. We also compare the result with the network performance theory as described in Section 4. For simulation, we used Matlab with network model as follows. All nodes $(N)$ in the network are uniformly distributed over an area of side length $2 S$ and has equal transmission range, $T_{r}$. We set $S=1000 \mathrm{~m}$ and $T_{r}=100 \mathrm{~m}$.

The node density is $\partial=N /(2 S)^{2}$ per $m^{2}$, and the average number of neighbors is $n=\partial \pi T_{r}^{2}-1$. We evaluated the throughput performance with various numbers of node and velocity of the node. Maximum node speeds were $2 \mathrm{~m} / \mathrm{s}, 4 \mathrm{~m} / \mathrm{s}, 6 \mathrm{~m} / \mathrm{s}, 8 \mathrm{~m} / \mathrm{s}$ and $10 \mathrm{~m} / \mathrm{s}$ and numbers of nodes were $20,25,30,35,40,45$ and 50, respectively. The environment used Rician with completely random channel [21], $K=4$ and Rayleigh fading propagation model. We used mobile-to-mobile channel model as described in [23]-[25]. Doppler frequency is affected by the transmitter and receiver movements, where in this simulation we used $f_{0}=2.4 \mathrm{GHz}$ and $c=3 \times 10^{8}$. The data packets were 512 bytes, transmitted at a rate of 10 packets per second, and channel bandwidth $2 \mathrm{Mb} / \mathrm{s}$.

Figure 6 shows the average throughput performance of conventional routing-TCP, routing-TCP theory, RTCP-RA, and RTCP-SNR with increasing number of nodes in Rician environment. Larger network, with increased node density will decrease the throughput in each node. From the result, the proposed method RTCP-SNR maintained a higher average throughput compared to the conventional routing-TCP and RTCPRA. The average throughput RTCP-SNR at 20 nodes density has advantages about $58.83 \%$ and $24.14 \%$ higher compared to conventional routing-TCP and RTCP-RA, respectively. At 50 nodes, in a higher density network, RTCP-SNR gained improvement about $50 \%$ and $23 \%$ compared to routing-TCP and RTCP-RA, respectively. The average throughput in Routing-TCP theory has the same semblance with the routing-TCP, this result complies with the analytical model approach, as described in Section 4. 


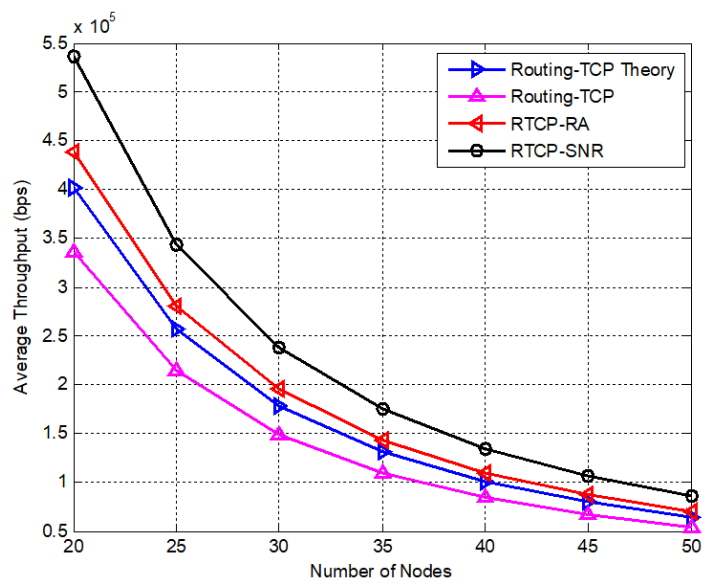

Figure 6. Average throughput Routing-TCP, Routing-TCP theory, RTCP-RA and Routing-TCP-SNR (RTCP-SNR) with numbers of nodes in Rician $K=4$

In Rayleigh fading environment, the network performance getting worse rather in Rician. This performance degradation occurred because in Rayleigh, the signal received by the receiver will vary rapidly and superposition of the overall amount of the reflected signal took place due to multipath. The average throughput of the proposed method RTCP-SNR outperformed the conventional routing-TCP and RTCP-RA as shown in Figure 7. For example, in smaller networks with number of nodes 20, RTCP-SNR can gain advantages about $72 \%$ higher than RTCP-RA and had almost twice significant improvement compare to the conventional routing-TCP. In larger network, with 50 nodes, the improvement was about $69 \%$ better than RTCP-RA. In Rayleigh environment, where bit error rate and the probability of misperception loss indication are high, the proposed method can deal with the situation more effectively than RTCP-RA. The average throughput Routing-TCP theory in Rayleigh had the same semblance with the routing-TCP that complies with the analytical model approach. In networks with higher density of nodes will increase the average number of neighbors. This situation produced a path with higher number of link connection and lower probability of success in sending the data as mention in Equation (14). An increasing number of link connection induced higher path break probability. RTCP-SNR maintains link in a path with better SNR connection than RTCP-RA and achive higher throughput.

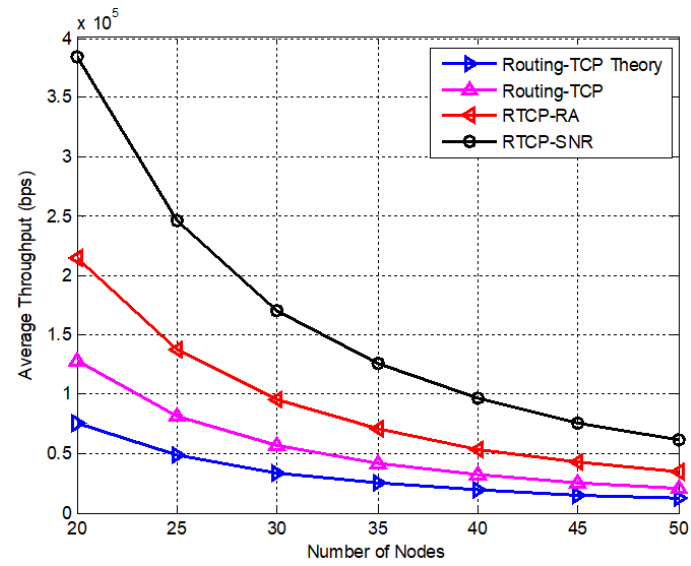

Figure 7. Average throughput Routing-TCP, Routing-TCP theory, RTCP-RA and Routing-TCP-SNR (RTCP-SNR) with increases number of nodes in Rayleigh fading

RTCP-SNR can gain higher performance with better SNR connection in the routing and minimize false congestion control when a loss indication occur. We analyze that better SNR connection can gain advantages in minimizing the average probability of error, as mentioned in Equations (20) and (25). 
The proposed algorithm in congestion control also prevents the situation when a loss indication ending as TDP is TO, due to both ways is unequal channel quality cause ACK is loss, by adjusting the current congestion window to get the maximum $E[\sigma]$, as describe in Equation (28). We observed that the gap between routing-TCP theory and the simulation routing-TCP was resulted because not all parameters in another layer mechanism were include in our cross layer scheme.

We compared the RTCP-SNR performance with routing-TCP as well the routing-TCP theory from Equation (13) in various of the velocity of nodes. Figure 8 shows the average throughput performance, where RTCP-SNR outperforms the conventional routing-TCP and RTCP-RA. We observed that RTCP-SNR can gain significant performance increases about $39.7 \%$ and $25 \%$ at velocity of node $2 \mathrm{~m} / \mathrm{s}$ compare to routingTCP and RTCP-RA, respectively. At velocity of nodes $10 \mathrm{~m} / \mathrm{s}$, the proposed method has gain advantages about $38.5 \%$ and $20 \%$ compare to routing-TCP and RTCP-RA.

In Rayleigh fading environment with various velocity scenarios, the network performance showed significant degradation. Throughput decreased as the node velocity increased. Figure 9 shows the average throughput Routing-TCP, Routing-TCP theory, RTCP-RA and RTCP-SNR with various velocity of nodes in Rayleigh fading. The proposed method RTCP-SNR had significant advantages and outperformed the conventional routing-TCP and RTCP-RA. At $2 \mathrm{~m} / \mathrm{s}$, RTCP-SNR gain improvements about 84\% improvement compared to RTCP-RA and almost one and a half times better than the conventional routingTCP. In higher speed of the nodes, at $10 \mathrm{~m} / \mathrm{s}$ can gained advatages about $70 \%$ compared to RTCP-RA.

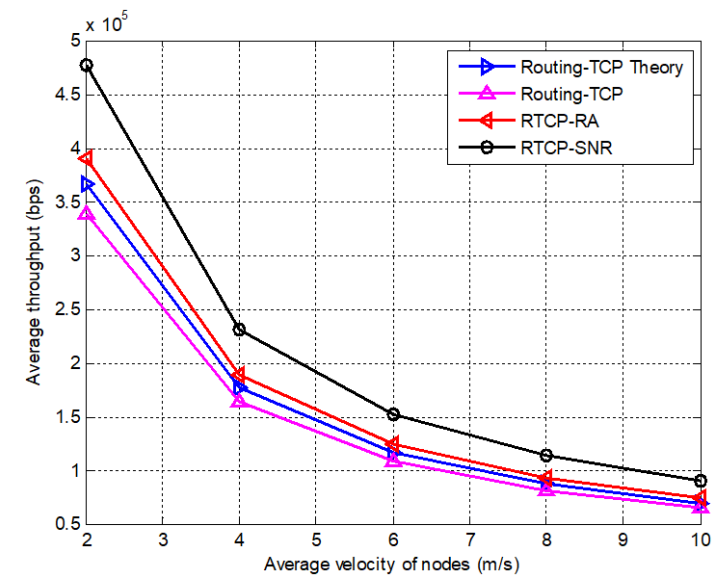

Figure 8. Average throughput Routing-TCP, Routing-TCP theory, RTCP-RA and Routing-TCPSNR (RTCP-SNR) with various velocity of nodes in Rician $K=4$

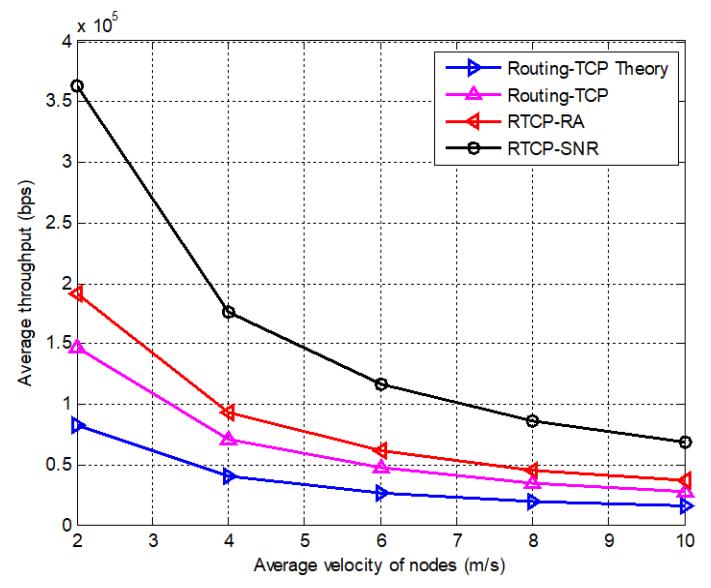

Figure 9. Average throughput Routing-TCP, Routing-TCP theory, RTCP-RA and Routing-TCPSNR (RTCP-SNR) with various velocity of nodes in Rayleigh fading

In a multipath fading environment such as Rician and Rayleigh, the velocity of the nodes has significant influence on network performance. Based on mobile-to-mobile channel model [23], [24], we analyzed the quality channel to node mobility and fading by using the crossing level rate $\left(N_{p}\right)$, namely the rate at which the signal envelope crosses a specified signal level $R_{t h}$. Higher level crossing rate indicates deteriorating channel quality with high bit error rate. In this model, $\rho=R_{t h} / R_{r m s}$ is the ratio between the transmission threshold $\left(R_{t h}\right)$ to the root mean square power of the received signal or the average $\operatorname{SNR}\left(R_{r m s}\right)$, factor $K$ shows the severity of the fading as describe in Equation (3), and $\alpha=V_{R} / V_{T}$ is the ratio between receiver and transmitter node velocities, then we can calculate the crossing level rate by:

$$
N_{p}=f_{D} \sqrt{2 \pi(K+1)} \rho e^{-K-(K+1) \rho^{2}} \sqrt{1+\alpha^{2}},
$$

If $f_{D}=f_{o} V_{T} / c$ is the maximum Doppler shift of the transmitter, where $f_{o}$ is the transmitter signal carrier frequency and $c=3 \times 10^{8} \mathrm{~m} / \mathrm{s}$ is the signal speed, then Equation (29) can be expressed as:

$$
N_{p}=\frac{f_{o}}{c} \sqrt{2 \pi(K+1)} \rho e^{-K-(K+1) \rho^{2}} \sqrt{{V_{T}^{2}+V_{R}^{2}}^{2}}
$$


From Equation (30), increasing node velocity will be increase the level crossing rate, and low $\rho$ will decrease the crossing level rate. For Rayleigh fading environment, $K=0$. With this approach, the higher average SNR connection will gain lower $\rho$ factor as the resulting decrease the level crossing rate. The algorithm in RTCP-SNR can reach higher SNR connections in the path and decrease the probability of error with lower of level crossing rate than the conventional routing-TCP and RTCP-RA.

In summary, RTCP-SNR outperformed the conventional routing-TCP and RTCP-RA, because it gained more throughputs with better algorithm mechanism as described in Sections 4. The proposed scheme can also improve the route selection process based on channel quality, SNR, and makes the routing protocol more adaptive in the multipath environment. Moreover, RTCP-SNR can reduce false congestion control when a loss indication as TDP is TO by adjusting the current congestion window, where the conventional routing-TCP cannot solve.

\section{CONCLUSION}

Since routing and congestion control algorithm have the risk of performance degradation due to random variation in channel condition, especially in Rician and Rayleigh environment. This paper has developed a detailed analytical routing and congestion control TCP throughput performance in Rician and Rayleigh environment, respectively. This analytical model is based on SNR with cross layer scheme. Through this analytical approach, we proposed joint routing and congestion control TCP model based on SNR (RTCP-SNR) mechanism. In this scheme, routing protocol will select the path based on highest SNR threshold $\left(\gamma_{t h}\right)$. When in high bit error rate and channel quality degradation, RTCP-SNR made adjustments on current congestion window based on SNR to prevent and to reduce a loss indication misperception, especially a loss indication in TDP is a TO. From the evaluation result RTCP-SNR outperformed the conventional routing-TCP, RTCP-RA, and had semblance with the theory. RTCP-SNR can gain high advantages with channel awareness in routing and congestion control TCP mechanism. For future work, the cross layer scheme in routing and congestion control TCP should consider application independence and application dependence.

\section{ACKNOWLEDGEMENTS}

This work is supported by Ministry of Research Technology and Higher Education of the Republic of Indonesia, Telkom University, and Yayasan Pendidikan Telkom.

\section{REFERENCES}

[1] R. Edirisinghe and A. Zaslavsky, "Cross-layer contextual interactions in wireless networks," IEEE Communications Surveys \& Tutorials, vol. 16, no. 2, pp. 1114-1134, 2014.

[2] A. Zhou, et al., "Joint traffic splitting, rate control, routing, and scheduling algorithm for maximizing network utility in wireless mesh networks," IEEE Transactions on Vehicular Technology, vol. 65, no. 4, pp. 2688-2702, 2016.

[3] Istikmal, et al., "Selective Route Based on SNR with Cross-Layer Scheme in Wireless Ad Hoc Network," Journal of Computer Networks and Communications, vol. 2017, 2017.

[4] G. Karbaschi and A. Fladenmuller, "A link-quality and congestion-aware cross layer metric for multi-hop wireless routing," IEEE International Conference on Mobile Adhoc and Sensor Systems Conference, Washington, DC, pp. 7, $655,2005$.

[5] L. Qu, et al., "Congestion Control, Routing, and Scheduling in Wireless Networks with Interference Cancelation Capabilities," in IEEE Transactions on Vehicular Technology, vol. 64, no. 7, pp. 3108-3119, 2015.

[6] E. Stai, et al., "Performance-Aware Cross-Layer Design in Wireless Multihop Networks Via a Weighted Backpressure Approach," in IEEE/ACM Transactions on Networking, vol. 24, no. 1, pp. 245-258, 2016.

[7] J. H. Choi and C. Yoo, "TCP-aware source routing in mobile ad hoc networks," Proceedings of the Eighth IEEE Symposium on Computers and Communications. ISCC 2003, vol. 1, pp. 69-74, 2003.

[8] A. T. Rahem, et al., "Node Cooperation to Avoid Early Congestion Detection Based on Cross-Layer for Wireless Ad Hoc Networks," International Journal of Electrical and Computer Engineering (IJECE), vol. 6, no. 5, pp. 2322-2330, 2016.

[9] Y. Wei, et al., "Cross-Layer Design for TCP Throughput Optimization in Cooperative Relaying Networks," 2010 IEEE International Conference on Communications, Cape Town, pp. 1-5, 2010.

[10] H. K. Molia and R. Agrawal, "A comprehensive study of cross - layer approaches for improving TCP performance in wireless networks," 2015 International Conference on Computing and Communications Technologies (ICCCT), Chennai, pp. 362-367, 2015.

[11] R. N. Devikar, et al., "Issues in Routing Mechanism for Packets Forwarding: A Survey," International Journal of Electrical and Computer Engineering (IJECE), vol. 6, pp. 421-430, 2016. 
[12] S. Ningning, et al., "Routing Algorithm for DTN Based on Congestion Control," TELKOMNIKA Telecommunication Computing Electronics and Control, vol. 11, no. 10, pp. 5617-5626, 2013.

[13] I. Vijaya, et al., "Influence of Routing Protocols in Performance of Wireless Mobile Adhoc Network," 2011 Second International Conference on Emerging Applications of Information Technology, Kolkata, pp. 340-344, 2011.

[14] C. E. Perkins and E. M. Royer, "Ad-hoc on-demand distance vector routing," Mobile Computing Systems and Applications, 1999. Proceedings. WMCSA '99. Second IEEE Workshop on, New Orleans, LA, pp. 90-100, 1999.

[15] U. Ahmad, et al., "Fairness Evaluation and Comparison of Current Congestion Control Techniques," Indonesian Journal of Electrical Engineering and Computer Science (IJEECS), vol. 1, no. 1, pp. 176-181, 2016.

[16] T. K. Sarkar, et al., "A survey of various propagation models for mobile communication," in IEEE Antennas and Propagation Magazine, vol. 45, no. 3, pp. 51-82, 2003.

[17] A. Goldsmith, "Wireless Communications," Cambridge University Press, pp. 644, 2005.

[18] J. Padhye, et al., "Modeling TCP Reno performance: a simple model and its empirical validation," in IEEE/ACM Transactions on Networking, vol. 8, no. 2, pp. 133-145, 2000.

[19] J. D. Spragins, et al., "Telecommunications protocols and design," 2006.

[20] K. D. Rao, "Channel Coding Techniques for Wireless Communications," Springer, XXI, pp. 255, 2015.

[21] W. Lindsey, "Error probabilities for Rician fading multichannel reception of binary andn-ary signals," in IEEE Transactions on Information Theory, vol. 10, no. 4, pp. 339-350, 1964.

[22] B. Sklar, "Rayleigh Fading Channels in Mobile Digital Communication Systems Part I: Characterization," IEEE Communications Magazine, 1997.

[23] A. S. Akki, "Statistical properties of mobile-to-mobile land communication channels," in IEEE Transactions on Vehicular Technology, vol. 43, no. 4, pp. 826-831, 1994.

[24] X. Chen, et al., "Channel-aware routing in MANETs with route handoff," IEEE Transactions on Mobile Computing, vol. 10, no. 1, pp. 108-121, 2011.

[25] R. J. Punnoose, et al., "Efficient simulation of Ricean fading within a packet simulator," Vehicular Technology Conference Fall 2000. IEEE VTS Fall VTC2000. 52nd Vehicular Technology Conference (Cat. No.00CH37152), Boston, MA, vol. 2, pp. 764-767, 2000.

\section{BIOGRAPHIES OF AUTHORS}

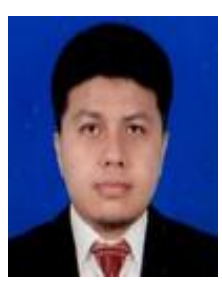

Istikmal is currently a Ph.D candidate at Institut Teknologi Bandung (ITB), the School of Electrical Engineering and Informatics. He received the B.E. degree in Electrical Engineering from STT Telkom, Indonesia, and M.E degree in Telecommunication Engineering, from Institut Teknologi Bandung (ITB). His interests include wireless mobile ad-hoc network, routing protocol, transport protocol, cross layer, telecommunication systems and computer. He is faculty staff in Telecommunication Engineering, School of Electrical Engineering, Telkom University.

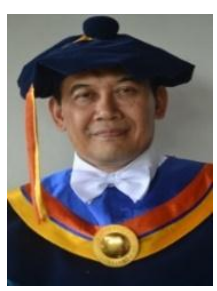

Adit Kurniawan is currently professor in School of Electrical Engineering and Informatics at Institut Teknologi Bandung (ITB), Indonesia. He received his B. E in Electrical Engineering from ITB, Indonesia, the M. Eng in Telecommunication Engineering from Royal Melbourne Institute of Technology, Australia, and the Ph.D in Telecommunication Engineering from University of South Australia. His fields of expertise are antenna and radio wave propagation, spread spectrum and CDMA communication.

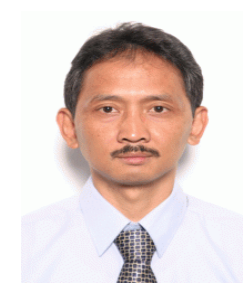

Hendrawan is currently lecturer in School of Electrical Engineering and Informatics at Institut Teknologi Bandung (ITB). He holds a B. E in Electrical Engineering from ITB, Indonesia, an M.Sc. in Telecommunication and Information Systems from University of Essex, UK, and a Ph.D in Electronic System Engineering from University of Essex, UK. His areas of interest include protocol communication, network performance and telematics. 